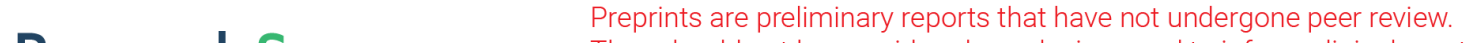 \\ They should not be considered conclusive, used to inform clinical practice, or referenced by the media as validated information. \\ Optothermophoretic flipping method for biomolecule interaction enhancement in biosensing
}

Jiajie Chen ( $\nabla$ cjj@szu.edu.cn )

Shenzhen University https://orcid.org/0000-0002-7200-6626

Youjun Zeng

Shenzhen University

Jie Zhou

Shenzhen University

Xueliang Wang

Shenzhen University

Ruibiao Miyan

Shenzhen University

Teliang Zhang

Shenzhen University

Wei Sang

Shenzhen University

Ying Wang

First Medical Center of PLA General Hospital

Haixia Qiu

Chinese PLA General Hospital

Junle Qu

Shenzhen University https://orcid.org/0000-0001-7833-4711

Ho-Pui Ho

The Chinese University of Hong Kong

Bruce Gao

Clemson University

\section{Yonghong Shao}

Shenzhen University

Ying Gu

Chinese PLA General Hospital

Article

Keywords: lab-on-a-chip, optothermophoretic flipping (OTF), surface plasmon resonance imaging (SPRi) 
Posted Date: March 17th, 2021

DOl: https://doi.org/10.21203/rs.3.rs-253405/v1

License: (c) (i) This work is licensed under a Creative Commons Attribution 4.0 International License. Read Full License

Version of Record: A version of this preprint was published at Biosensors and Bioelectronics on May 15th, 2022. See the published version at https://doi.org/10.1016/j.bios.2022.114084. 


\title{
Optothermophoretic flipping method for biomolecule interaction enhancement in biosensing
}

\author{
Jiajie Chen ${ }^{\text {a, 1, }}$ Y Youjun Zeng ${ }^{\mathrm{a}, 1}$, Jie Zhou ${ }^{\mathrm{a}}$, Xueliang Wang ${ }^{\mathrm{a}}$, Ruibiao Miyan ${ }^{\mathrm{a}}$, Teliang Zhang ${ }^{\mathrm{a}}$, Wei Sang ${ }^{\mathrm{a}}$, Ying \\ Wang ${ }^{\mathrm{b}}$, Haixia Qiu ${ }^{\mathrm{b}}$, Junle $\mathrm{Qu}^{\mathrm{a}}$, Ho-Pui $\mathrm{Ho}^{\mathrm{c}}$, Bruce Zhi Gao ${ }^{\mathrm{d}}$, and Yonghong Shao ${ }^{\mathrm{a},}$, , Ying $\mathrm{Gu}^{\mathrm{c}}$

\begin{abstract}
${ }^{a}$ Key Laboratory of Optoelectronic Devices and Systems of Ministry of Education and Guangdong Province, College of Physics and Optoelectronics Engineering, Shenzhen University, Shenzhen 518060, China

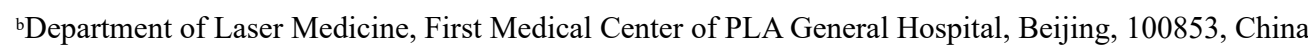

'Department of Biomedical Engineering, The Chinese University of Hong Kong, Shatin, Hong Kong

${ }^{\mathrm{d} D e p a r t m e n t}$ of Bioengineering and COMSET, Clemson University, Clemson, SC 29634, US

${ }^{1}$ These authors contributed equal to the article.

* Corresponding authors. E-mail addresses: cij@szu.edu.cn (J. Chen), shaoyh@szu.edu.cn (Y. Shao).
\end{abstract}

\begin{abstract}
The widely used surface-based biomolecule sensing scheme has greatly facilitated the investigation of protein-protein interactions in lab-on-a-chip microfluidic systems. However, in most biosensing schemes, the interactions are driven in a passive way: The overall sensing time and sensitivity are totally dependent on the Brownian diffusion process, which has greatly hindered their efficiency, especially at low concentration level or single-molecule analysis. To break this limitation, we developed an all-optical active method termed optothermophoretic flipping (OTF). The biomolecules were first enriched to aggregation and then pushed to their counterparts for effective contact via a flipped thermophoresis. As a proof-of-concept experiment, we tested its performance via antibody-antigen binding on a surface plasmon resonance imaging (SPRi) platform. We achieved 36.9-fold sensitivity enhancement in this first temporal modulated approach that manipulates biomolecules for interaction enhancement. This method promises to be widely adopted in various biosensing platforms that require ultrasensitivity in colloidal sciences and biochemical studies.
\end{abstract}

\section{Introduction}

Proteins are vital components in a living organism; they play an important role in energy transportation, cell functioning, and immune response. The study of protein-protein interactions is a hot topic in the field of biosensing. Especially, the antibody and antigen interactions are of great importance in point-of-care diagnosis. ${ }^{1,2}$ Numerous biosensing schemes such as enzymelinked immunosorbent assays (ELISA), ${ }^{3-5}$ Colorimetric assays, ${ }^{6}$ material fluorescence, ${ }^{7-9}$ chemiluminescence assays, ${ }^{10,11}$ Raman spectroscopy, ${ }^{12-15}$ and surface plasmon resonance (SPR) ${ }^{16,17}$ biosensors have been developed to investigate the corresponding biochemical properties and behaviors. Nevertheless, most of the biomolecular sensors are designed to act passively, i.e., the driving force for an effective interaction between target biomolecules is mainly dependent on their 
Brownian motion and diffusion process. Although some microstructures are designed to enhance the sensing event, ${ }^{18,19}$ at low sample concentration level, the possibility of an effective collision for subsequent biomolecule binding becomes quite low, which inhibits subsequent sensing performance.

In the past decade, various kinds of active biosensing techniques emerged. Generally, these schemes are primarily of two types: One is nanostructures with precise fabrication processes, and the other is the method to drive and enrich the solutes towards the vicinity of the sensing surface via different kinds of active external forces. For example, in 2011, a DNA sensing enhancement scheme was developed using super hydrophobic nanostructures for solute concentration; a sample of extremely low concentration (aM) can be detected after solvent evaporation. ${ }^{20}$ For liquid-based enhancement and manipulation schemes, acoustical, ${ }^{21-23}$ electrical, ${ }^{24-26}$ and optothermal effects, ${ }^{27-}$ ${ }^{31}$ are the three most commonly used methods.

Two aspects can serve as the standard to accessing the sensing enhancement level: One is sensing time reduction rate, and the other is the sensitivity increment rate. For example, an electrothermal induced swirl-like flow assisted antibody-antigen binding enhancement strategy is proposed and the authors have achieved a 10-fold sensing time reduction. ${ }^{24}$ In 2018, an electro-thermoplasmonic (ETP) effect enhancement scheme reached $2.9 \times$ faster than the diffusion-limited case. ${ }^{29}$ In 2020, Y. Kim et al proposed a bubble-mediated molecular concentration method to enrich the biomolecule to the border of the thermal induced bubble; they have achieved an sensitivity enhancement of $8.7 \times .{ }^{27}$ However, there is still much room for further sensitivity improvement especially for the protein-protein interactions. Currently, the major optothermal diffusion-limited breaking methods still need the assistance of other forces such as electrical field or multi-phase interactions. Moreover, in the majority of sensing enhancement strategies, the sensing signal is in 1D, i.e., an integrated signal from the sensing area which lacks effective imaging ability to monitor the whole $2 \mathrm{D}$ sensing area in real time.

To address the above issues, we present a surface plasmon resonance imaging (SPRi) based optothermophoretic flipping (OTF) method for biomolecule interaction enhancement, and we have greatly enlarged the sensitivity enhancement to a factor of 36.9. The SPRi is an emerging tool in recent years that offers label-free, real-time, and high sensitive sensing, and it can be extensively applied in biochemistry studies. ${ }^{32-36} \mathrm{Here}$, we adopt the wavelength based SPRi (WSPRi) as our sensing platform. ${ }^{37-39}$ Especially, the WSPRi can provide us a hyperspectral image of resonance wavelength of SPR, i.e., each pixel on the image of the sensing surface serves as an independent sensing unit to obtain its own SPR spectrum. This can provide not only intermolecular interaction information in multiple sensing sites, but also a high throughput of signal detection. ${ }^{40-42}$ In addition, the OTF method is based on thermophoretic force exerted on biomolecules or nanoparticles induced by temperature gradient, ${ }^{43-47}$ and the thermophoretic force has recently drawn great attention in the study of biological problems. ${ }^{28,46,48-52}$ Moreover, a recent study indicated that by adopting the plasmonic thermal effect, ${ }^{53}$ one can polish the traditional optical manipulation method to a more efficient and accurate level of sub- $10 \mathrm{~nm}$ biomolecules. ${ }^{30} \mathrm{In}$ this study, we designed an in-situ thermophoresis-driven molecular interaction enhancement strategy for lab-onchip applications. This, to our knowledge, is the first use of timely varying optothermophoresis for biomolecular manipulation and interaction enhancement. The sensitivity enhancement factor of 36.9 is the highest enhancement factor ever achieved in existing active SPR sensing schemes. The approach provided an active biosensing method that can be adopted in a wide range of sensing 
platforms. In addition, the combination of WSPRi technique here also enables $2 \mathrm{D}$ real-time imaging of the biomolecular binding event in the near-field region.

\section{Results}

\section{Experimental setup}

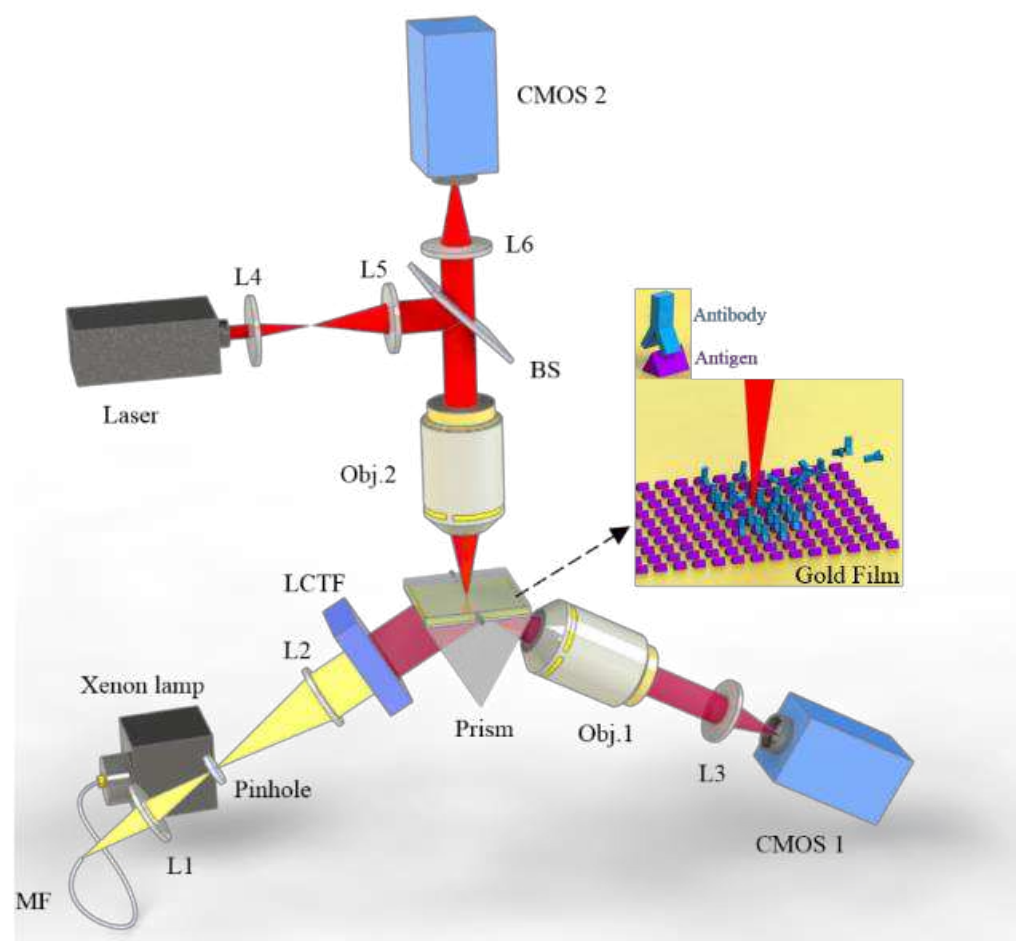

Fig. 1. The optical setup. The system consisted of two parts: WSPRi path and optothermal excitation path. L1-L6: Lens; Obj.1-2: Objects lens; MF: Multimode optical fiber; BS: Beam splitting.

\section{Wavelength based surface plasmon resonance imaging (WSPRi)}

A schematic of our setup is shown in Fig. 1. The system is designed into two parts: the WSPRi path below the sensor chip and the optothermal excitation part on top.

For the WSPRi path, in the left arm, a halogen lamp was used as the SPR excitation light source. The broadband-spectrum light from the halogen was coupled to a multimode optical fiber (MF) through a group of lenses. The fiber light passed through a collimating lens group (L1, L2) and a pinhole; the parallel light reached to the liquid crystal tunable filter (LCTF), which could filter out a narrow-band light with selected central wavelength. In the experiments, the wavelength scanning range was from $610 \mathrm{~nm}$ to $646 \mathrm{~nm}$ with a scanning step of $2 \mathrm{~nm}$. Then it was coupled into the prism and excites the surface plasmon wave on the surface of Au-coated sensing chip (Fabrication process is shown in Methods). To monitor the combination process of antigen and antibody binding (enlarge view in Fig. 1), in the right arm, the light with sample information, after reflection by the sensing region, passed through the microscopic imaging group consisting of objective lens (10X Nikon Plan Fluorite Imaging Objective, 0.3 NA), tube lens, and the CMOS1 (Imaging, DMK33GP1300) 
for subsequent SPR imaging. During the experiments, the WSPRi constantly monitored all the SPR spectra on the sensing surface, and each pixel on the image of the sensor surface could produce one SPR curve, which represented the molecule binding event in near-field of the sensing surface. Therefore, a whole SPR hyperspectral image was obtained in real time, and the resonance wavelength shift $\Delta \lambda$ gives us an indication about the association rate of the biomolecules on the sensing region. ${ }^{37}$

In addition, the optothermal excitation part was designed for molecular manipulation and enrichment. We employed a $785 \mathrm{~nm}$ laser (CNI, BE5116) as the heating source, controlled by a signal generator (Tektrinix, AFG1062). The laser beam was focused to a spot size of $3 \mu \mathrm{m}$ in diameter after passing through the expander lens group ( $L 4, L 5)$, beam splitter (BS), and the objective lens (10X Nikon Plan Fluorite Imaging Objective, $0.3 \mathrm{NA}$ ). Laser power was set to $60 \mathrm{~mW}$. An image sensor CMOS2 on the top (Imaging, DMK33GP1300) was for sample visualization during testing. Two kinds of optothermal induced thermodynamic phenomena, convective flow and thermophoresis, were excited for biomolecular enrichment and sensing. The two phenomena have greatly facilitated optical force induced tweezers ${ }^{54}$ because of their abilities of long-range manipulation and facile energy-efficient trapping of small particles in recent years. ${ }^{55-57}$

\section{Working principle}

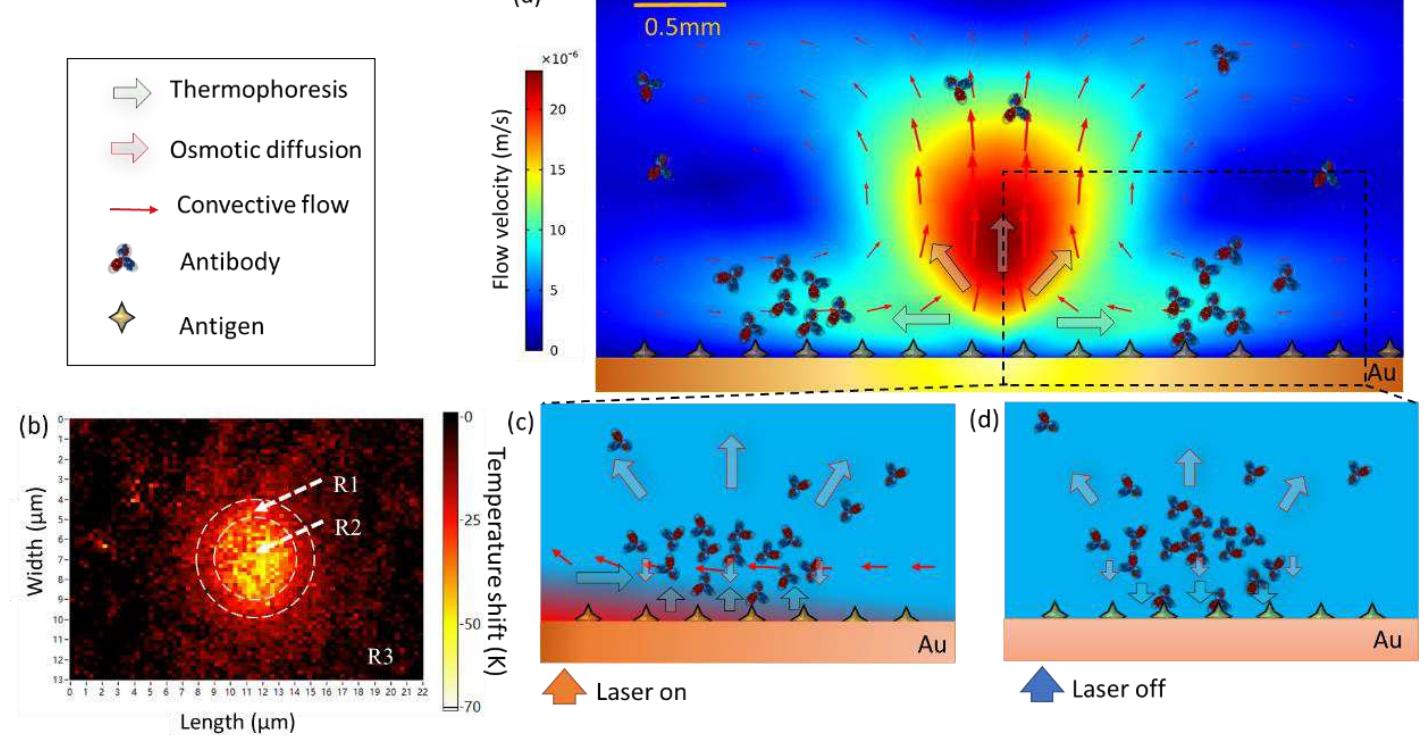

Fig. 2. Schematic illustration and theoretical analysis of OTF system. (a) Side view of the entire system when the heating laser is switching on. The convective flow and thermophoresis relocated and enriched the biomolecules from bulk solution to a ring shape region where the temperature is in relatively lower level; (b) Top view of the ring shape biomolecule aggregation region in the background of near-field temperature map. R1: Biomolecule enrichment and binding region; R2: heating laser focusing center region; R3: background region with no laser heating. (c) Zoom-in picture of the biomolecule enrichment region when laser is switched on, the upward thermophoretic (UT) force induces inefficient binding of the biomolecules; (d) Zoom-in picture of the biomolecule enrichment region when laser is just switched off, the flipped thermophoretic (FT) 
force (downward) induces efficient binding of the biomolecules.

The interaction between antibody and antigen is a complex and dynamic phenomenon based on noncovalent forces. The interaction between antibody $\mathrm{Ab}$ and antigen $\mathrm{Ag}$ at equilibrium can be expressed as: 58

$$
\mathrm{Ab}+\mathrm{Ag} \rightleftharpoons \mathrm{Ab}-\mathrm{Ag}
$$

When antibody $\mathrm{Ab}$ and antigen $\mathrm{Ag}$ tend to combine, several criteria are required: (i) The distance between $\mathrm{Ab}$ and $\mathrm{Ag}$ is small enough; (ii) The two molecules must be arranged in the proper spatial orientation for an effective binding. To promote the antibody and antigen binding event, we need to disrupt the original Brownian diffusion-limited dynamics and push the corresponding biomolecules to their counterparts to form an effective binding. Here, in the microfluidic channel with a low Reynolds number, we designed our system by simply adopting two laser excited thermodynamic forces. As the operation principle in Fig. 2 depicts, after switching on the heating laser of $785 \mathrm{~nm}$, the temperature profile and the convective flow are generated, and they can be stabilized in about $1 \mu \mathrm{s}$ after laser excitation. ${ }^{59}$ There exist two dominate thermodynamic phenomena; one is large scale convective flow, and the other is temperature gradient dependent thermophoresis. As the red arrow in Fig. 2(a) shows, in the microfluidic channel, the convective flow brings biomolecules from hundreds of micrometers away to the hottest region in a toroidal shape which is governed by Navier-Stokes equations, and the convective force is dominated by Stokes' law:

$F_{C O N}=6 \pi \eta R v$

Where $\eta$ is the viscosity coefficient, $R$ is the hydraulic radius of the suspended antibody, and $v$ is the relative velocity between the antibody and the fluid. Therefore, the biomolecules suspended in the solution are migrating in a toroidal shaped convective flow cycle. Note that all the solute and water molecules are migrating together with the biomolecules in the cycle. However, when some of the biomolecules approach the region with a higher temperature gradient (near the flow convergence center), the thermophoretic force $\left(F_{T H}\right)$ exerted on the molecules plays the major role. Thermophoresis, which describes the drift of particles or molecules due to the temperature gradient in the solvent, is usually described by the formula:

$v_{T}=-D_{T} \cdot \nabla \mathrm{T}$

Where, $v_{T}$ is the driving velocity of microparticles or biomacromolecules in the solution, $\nabla \mathrm{T}$ is the temperature gradient, $D_{T}=S_{T} \cdot D$ is the thermophoresis mobility, $S_{T}$ is the Soret coefficient and $D$ is the diffusion coefficient induced by Brownian motion. Although the formula (2) seems quite simple, the magnitude and direction of $D_{T}$ or $S_{T}$ are susceptible to many parameters in the surrounding solutions and the biomolecule itself, such as the molecule's hydraulic size, ${ }^{60}$ surface entropy, ${ }^{61,62}$ surface hydrophilicity, ${ }^{44}$ surface charge, ${ }^{47,63-65}$ and solution ion composition and $\mathrm{pH}$ value. ${ }^{43,45,61}$

In the electrolyte solution, the thermophoretic force $F_{T H}$ on the charged biomacromolecules governs by thermo-electrophoresis mechanism which known as the Seebeck effect. ${ }^{47}$ Analog to the PN junction charge diffusion, the temperature gradient can introduce redistribution of anions and cations in the solution and thus can produce a thermal-induced internal electric field that can drive the charged particles or macromolecules. A recent study gives a summary and explanation of the mechanism via molecular dynamics simulations; it indicates that a particle with negative surface charge will be driven to a cooler region in an inorganic electrolyte solution such as sodium 
chloride $(\mathrm{NaCl}) .65$

Typically, a PBS solution (phosphate buffered saline, $\mathrm{pH}$ 7.4) is the most used buffer in biomedical experiments. The anions and cations in the solution are majorly $\mathrm{Cl}^{-}$and $\mathrm{Na}^{+}$(The $\mathrm{NaCL}$ is $136 \mathrm{mM}$ in commonly used $0.1 \mathrm{M}$ PBS solution) as well as a small amount of phosphate. At $\mathrm{pH}=7.4$ the surface charge of most proteins, such as Immunoglobulin $\mathrm{G}$ (IgG) proteins, is negative (represented by Debye-Hückel-Henry charge, $Z_{D H H}$ is ranged from 0 to -13$), 66,67$ which gives a positive $S_{T}$; therefore according to Equation 2, the macromolecules such as IgG protein are thermophobic and will be pushed away from the hottest laser spot center. In the steady state, this thermophoresis driven molecular flow is balanced by the convective flow and ordinary mass diffusion; a ring-shape steady-state concentration profile (R1 in Fig. 2(b)) is established in the vicinity of the gold film(Au film), which is similar to a DNA thermal enrichment scheme with positive $S_{T}$. ${ }^{68}$ The measured near-field temperature rising in this region R1 is about 20K; the positive $S_{T}$ prevents the biomolecules from going into the laser focusing center region R2 which gives the highest temperature rising.

Nevertheless, in this scenario, although the molecules (antibody) are gathered near the Au film, they cannot efficiently bind with the antigen on the Au film. Since the Au film and the glass substrate have a much higher thermal conductivity and lower specific heat capacity than the bulk solution, the temperature of the Au film $\left(T_{A u}\right)$ is higher than that of the surrounding solution $\left(T_{S o}\right)$; our simulation indicates that their temperature difference $\Delta T=T_{A u}-T_{S o}$ is about $5 \mathrm{~K}$ [Fig.3(d)]. Therefore, as the force analysis in Fig. 3(a) indicates, the aggregated molecules experience a thermophoretic force (vertical component of $F_{T H}$ ) in the upward direction that tends to push them away from the Au film. And at a certain radial distance near the laser spot edge, $F_{T H}$ is counterbalanced by the convective dragging force $F_{C O N}$ and the particle-surface interaction force $F_{P S}{ }^{69,70}$ Therefore, at the steady state when the laser is switching on, the antibodies can aggregate in a stagnant ring shaped zone where the net force is zero in the vicinity of the Au film. However, because the upward $F_{T H}$ becomes larger when the proteins are getting close to the Au surface, the proteins cannot efficiently bind with the antigens on the gold surface at this stage. 
(a)

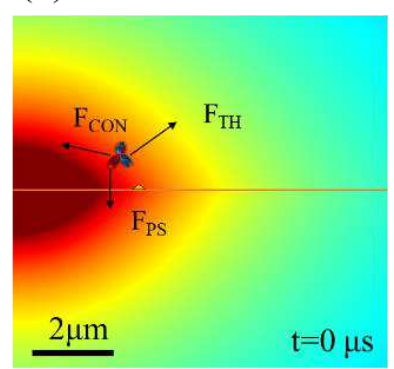

(d)

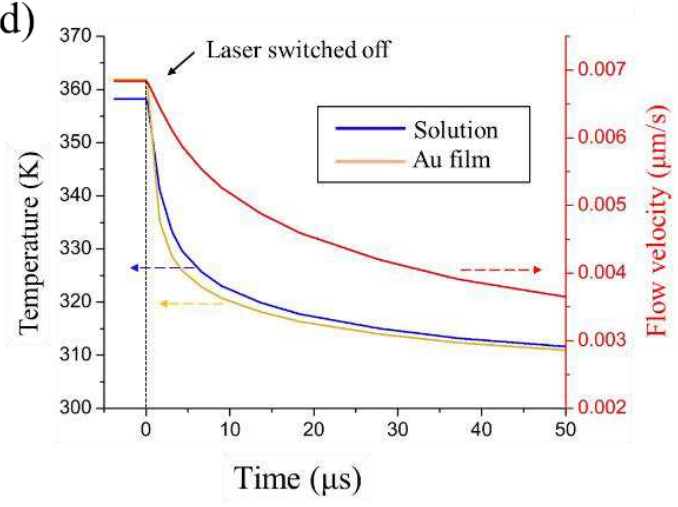

(c)
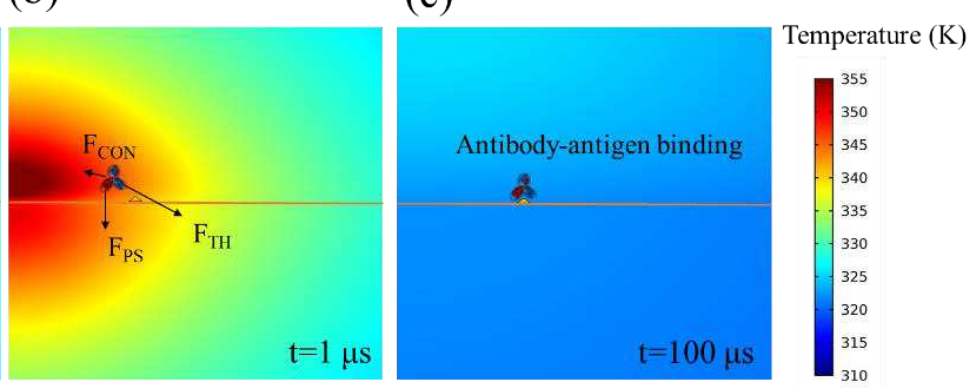

(e)

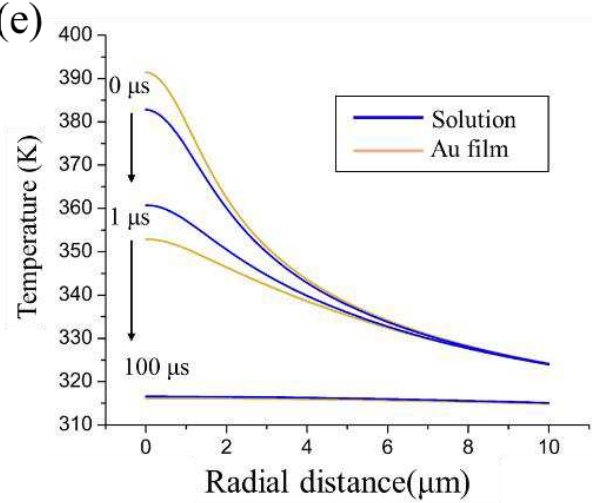

Fig. 3. Temperature distribution and force analysis in OTF antibody-antigen binding enhancement method. (a)-(c) Temperature distribution (symmetrical half) near the laser spot at $0 \mu$ s (heating laser switched off), $1 \mu \mathrm{s}$, and $100 \mu \mathrm{s} . F_{C O N}, F_{T H}$, and $F_{P S}$ are convective drag force, thermophoretic force, and particle-surface interaction force respectively. (d) Temperature temporal variation comparison between the sample solution and the Au film surface as well as the convective flow velocity variation. The temperature and flow velocity interrogation point locate at $1.5 \mu \mathrm{m}$ radial distance from the laser spot center and $200 \mathrm{~nm}$ above the Au film. (e) Temperature radially distribution in $200 \mathrm{~nm}$ above the Au film.

On the contrary, when the laser is just switched off, a flipped thermophoretic force which greatly facilitates biomolecular binding is instantaneously generated. As shown in Fig. $3(\mathrm{~d}, \mathrm{e})$, from the very moment when the laser is switched off to a transient time about tens of microseconds afterwards, the heat dissipates more faster in the Au film than in the solution near the interface. The temperature in the bulk solution is higher than the Au film, so the $\Delta T$ is about $-4.5 \mathrm{~K}$. While the $S_{T}$ remains positive, so the vertical component of the thermophoretic force $F_{T H}$ is temporally reversed and pointing to the gold film, and the convective drag force $F_{C O N}$ becomes even smaller in the vicinity of the surface. (Fig. 3(b, d)) Therefore, accompany with the particle-surface interaction force $F_{P S}$ and osmotic diffusion induced by the concentration difference, the aggregated biomolecules near the Au film are pushed to the Au film, and a higher binding rate occurs at this transient time after the heating laser is switched off. Subsequently, at laser-off status

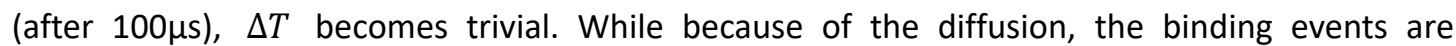
continuing until an equilibrium stage is reached when the concentration difference is smaller.

\section{Antibody-antigen binding experiments}

As a proof-of-concept experiments, we did an antibody-antigen binding test to assess the 
enhancement ability of our method. Here we used an antibody-antigen pair of human IgG/goatanti-human IgG. After a pretreatment process of the sensing chip (See method), a layer of human IgG probes was fixed on the Au film. Then $500 \mathrm{ng} / \mathrm{ml}$ goat-anti-human IgG solution (PBS buffering) was injected into the flow cell. And the binding event was monitored by the WSPRi. Note that in this level of molecule concentration, the corresponding $\Delta \lambda$ is not detectable in traditional WSPRi without the interaction enhancement. ${ }^{35,71}$ And the SPR wavelength shifting during the testing is presented in Fig. 4(a), 5 cycles of laser heating with on/off (120s/300s) modulation are applied on the Au film, i.e., in each cycle, the duration of laser switching on and off time was 120 s and 300s, respectively. The SPR wavelength shift $\Delta \lambda$ is in positive correlation to the solution's refractive index as well as the effective binding of the goat-anti-human IgG protein. ${ }^{35,42}$ And a rising temperature can decrease solution's refractive index. ${ }^{72}$ Therefore, the SPR wavelength dropped dramatically as the heating laser was switched on.

The experimental results were consistent with our simulation. After 5 cycles of the OTF-enhanced IgG protein binding, the whole sensing site could be divided into three regions (R1-R3) as Fig. 2(b) indicates. The region R3 without heating laser treatment has no SPR wavelength shift at each cycle, and the region R2 where the laser focusing center is located had insignificant SPR wavelength shift because of the upward thermophoretic force. While the region of R1, where proteins have the aggregation and efficient binding, produces the most obvious wavelength shift.

In addition, during the heating laser illumination in each cycle, the upward thermophoretic (UT) force precludes effective binding. So, the resonance wavelength shift $\Delta \lambda_{U T i}(i=1,2,3 \ldots)$ at laser-on status in each cycle was quite small. When the laser was switched off, the heat in micrometer scale dissipated rapidly. The transient flipped thermophoretic (FT) force is in a downward direction, which greatly promoting the binding events. Therefore, at each rising edge of the curve, the change of resonance wavelength $\Delta \lambda_{F T i}$ ( $\left.\mathrm{i}=1,2,3 \ldots\right)$ induced by $\mathrm{FT}$ force gives the largest $(1-2 \mathrm{~nm})$ value in the whole modulation cycle. Subsequently, during the time without heating laser illumination, the osmotic diffusion and Brownian motion induced diffusion governed the binding event, the corresponding change of resonance wavelength $\Delta \lambda_{D i}(\mathrm{i}=1,2,3 \ldots)$ was maintained at a lower level $(<1 \mathrm{~nm})$. Moreover, the WSPRi manifested its unique advantage of SPR wavelength imaging in this experiment; it has greatly facilitated finding the corresponding biomolecular binding region's location, shape and concentration. As the SPR wavelength image indicates (Fig. 4(b)), the goat-antihuman IgG proteins were aggregated in a ring-shape with a horizontal radius of $2.5 \mu \mathrm{m}$ from the heating laser focusing spot ( $3 \mu \mathrm{m}$ in diameter) center. 
(a)

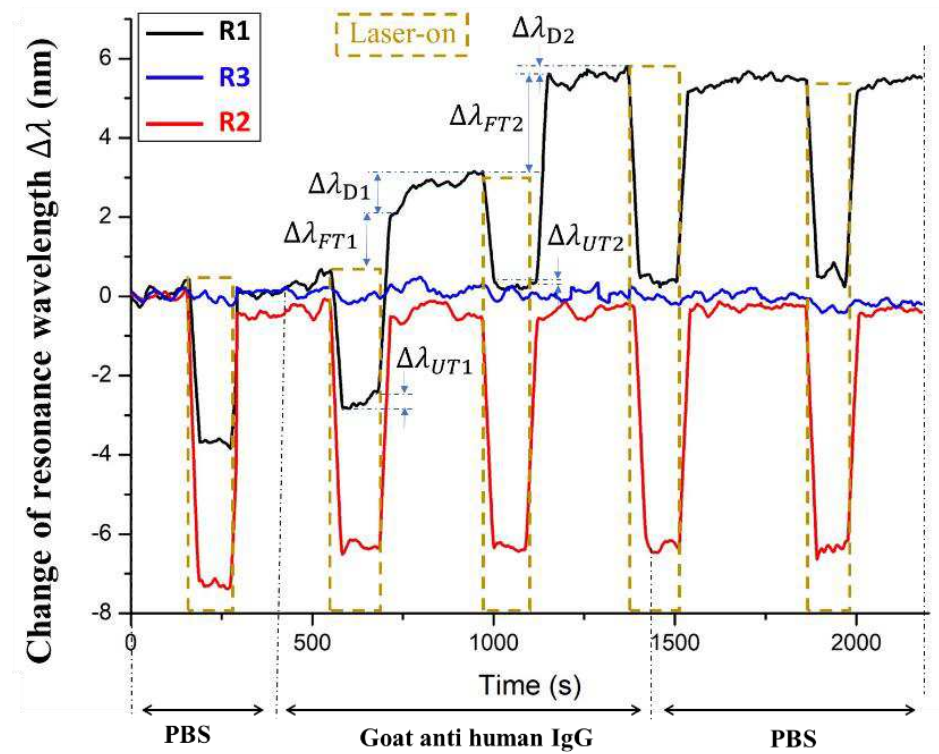

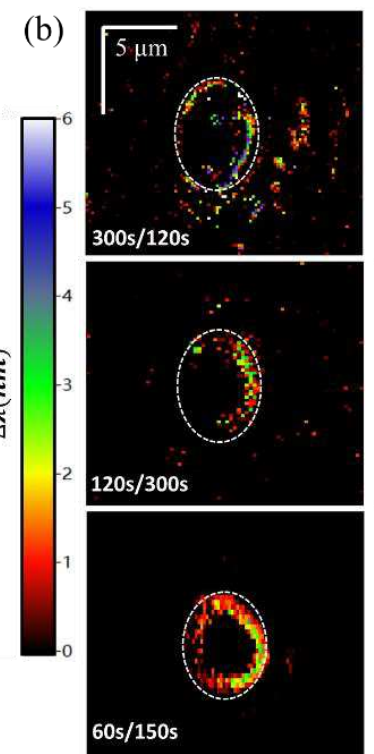

Fig. 4. Experimental demonstration of OTF-enhanced SPR imaging capabilities via binding of 0.5 $\mu \mathrm{g} / \mathrm{mL}$ goat anti human IgG with the probes on gold surface. (a) Temporal variation of the $\Delta \lambda$ induced by the heating laser on/off modulation (120s/300s on/off period). Three curves are the resonance wavelength real-time response curves, and each point on the curve is the averaged value in the corresponding sub-regions ( $4 \times 6$ pixels) that produce the largest resonance wavelength shift from the three interrogation regions of R1, R2 and R3 in IgG binding experiments. (b) SPR image of the whole sensing site of three on/off period of 300s/120s, 120s/300s, and 60s/120s, respectively. The change of resonance wavelength $\Delta \lambda$ indicates the SPR peak shifting after the experiment.

Moreover, although the highest binding rate was attributed to the falling edge of the heating laser modulation (laser switched off), the laser on/off duration matters, too. The modulation function can be further optimized to produce the highest biomolecular binding rate. We did a series of systematic experiments with different heating laser modulation functions. As shown in Fig. 5, the modulation functions have different duty cycles and periods. It turns out that the $120 \mathrm{~s} / 300 \mathrm{~s}$ on/off period can produce the highest binding rate. In the period of laser on or off, the localized concentration of the biomolecules in the solution will gradually reach to their equilibrium stage. When the laser is on, the UT force prevents an efficient binding, the molecules in the surrounding solution need some time to form a detectable biomolecule aggregation, so that the molecules in the vicinity of the Au film can be well prepared for binding. The results have shown that 120 s of laser-on time is enough for the biomolecules to form an efficient concentration enrichment region that reaches the dynamic balance. And a shorter laser-on time will induce lesser amount of molecule enrichment, and a longer time will make no further contribution to the molecule's enrichment as the equilibrium stage has already reached in 120 s.

Subsequently, after concentrating an enough number of molecules in the vicinity of Au film in laseron status, in the laser-off status, following the transient time of FT force vanishing, the binding event is governed by the osmotic force induced by the concentration gradient and the Brownianmotion diffusion. However, the diffusion is towards all directions. Therefore, it takes about 300 s to reach the equilibrium where the curve is at a plateau stage, and the $\Delta \lambda_{D i}$ reaches its largest value. 
Noted that a longer off-status duration could not give a larger $\Delta \lambda_{D i}$, the molecules will diffuse away from the efficient binding area, which hinders the re-formation of the molecule aggregation in next cycle's on-status duration. Therefore, the optimized on/off time of heating laser is $120 \mathrm{~s} / 300 \mathrm{~s}$ for getting a highest biomolecule binding rate at the laser power of $60 \mathrm{~mW}$. The optimized on/off time varies from different heating laser parameters such as its wavelength or optical power density as well as the biomolecular categories. The users can tune the laser modulation function and find the optimized on/off time based on experimental conditions. In addition, the resonance wavelength imaging capability also enables users to find the most sensitive subregion that produces the largest $\Delta \lambda$.

(a)

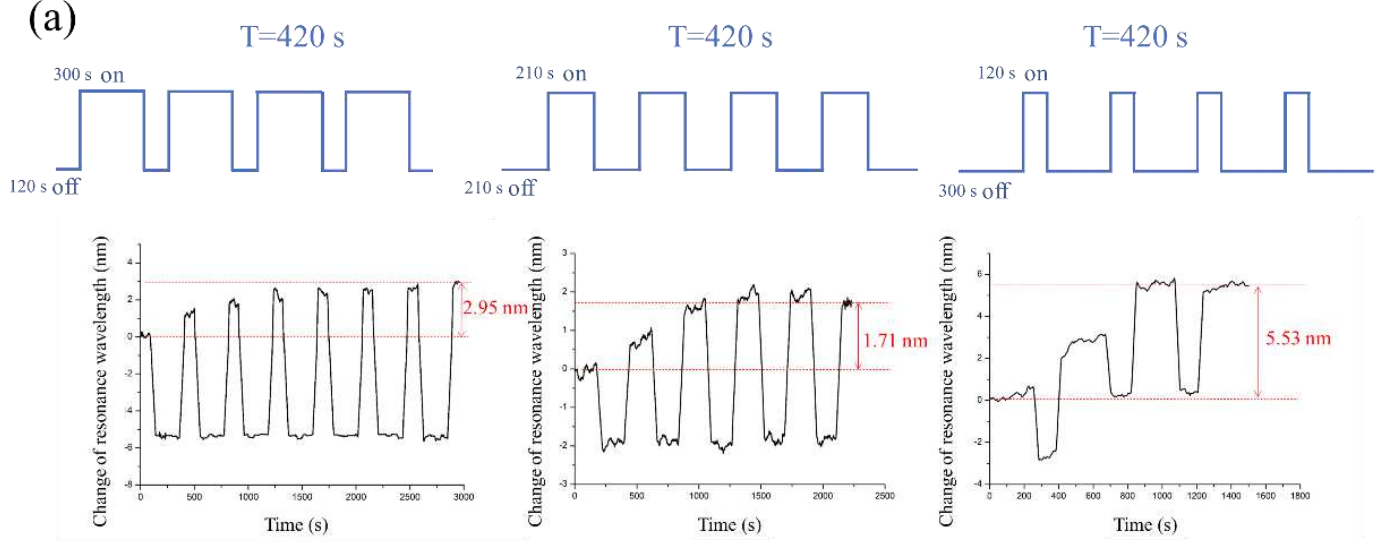

(b)

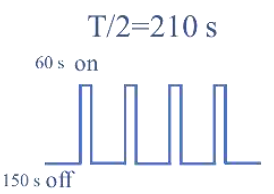

$\mathrm{T} / 3=140 \mathrm{~s}$

$1020 \mathrm{~s}>2.4 \mathrm{~T}$
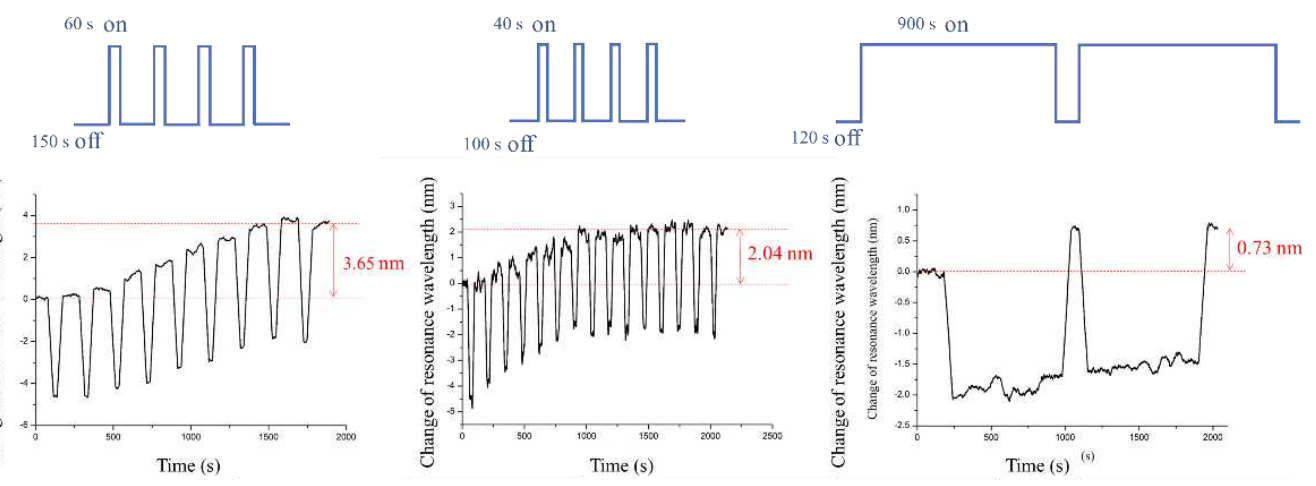

Fig. 5. Optimized heating laser on/off time investigation in goat anti human IgG $(500 \mathrm{~nm} / \mathrm{ml})$. Under the heating laser power of $60 \mathrm{~mW}$, a series of heating laser triggering signal (laser on/off duration) of different (a) duty cycles and (b) period T are studied. The net SPR wavelength shift after the experiments $\Delta \lambda$ is denoted in red. Each point on the curves is the averaged value of resonance wavelength change in the corresponding sub-regions of $4 \times 6$ pixels that produce the largest $\Delta \lambda$ from the ring-shape interrogation region $\mathrm{R} 1$.

In addition, we also tested the performance in different biomolecular concentrations. As Fig. 6 shows, a lower concentration produced a smaller $\Delta \lambda$. Simultaneously, in IgG concentration of $500 \mathrm{ng} / \mathrm{ml}$, the same system without OTF gave a $\Delta \lambda$ of $0.15 \mathrm{~nm}$. While when the OTF method is applied, the respective $\Delta \lambda$ is enhanced to $5.53 \mathrm{~nm}$. Therefore, the sensitivity enhancement factor of our system is about 36.9x which is by far the highest sensitivity enhancement factor for the wavelength scanning type SPR sensor. Moreover, the inter-biomolecular affinity varies differently 
in different categories; ${ }^{58}$ a higher association rate can produce a higher sensitivity and lower detection limit. Also, the adopted sensing platform of WSPRi is wavelength based, which cannot intrinsically produce the highest sensitivity. It is well known that the phase modes can produce the highest sensitivity among the four SPR interrogation methods of intensity, angle, wavelength and phase modes. ${ }^{36,73}$ Therefore, the sensitivity can be further improved if the OTF method is applied in other biosensing platforms.

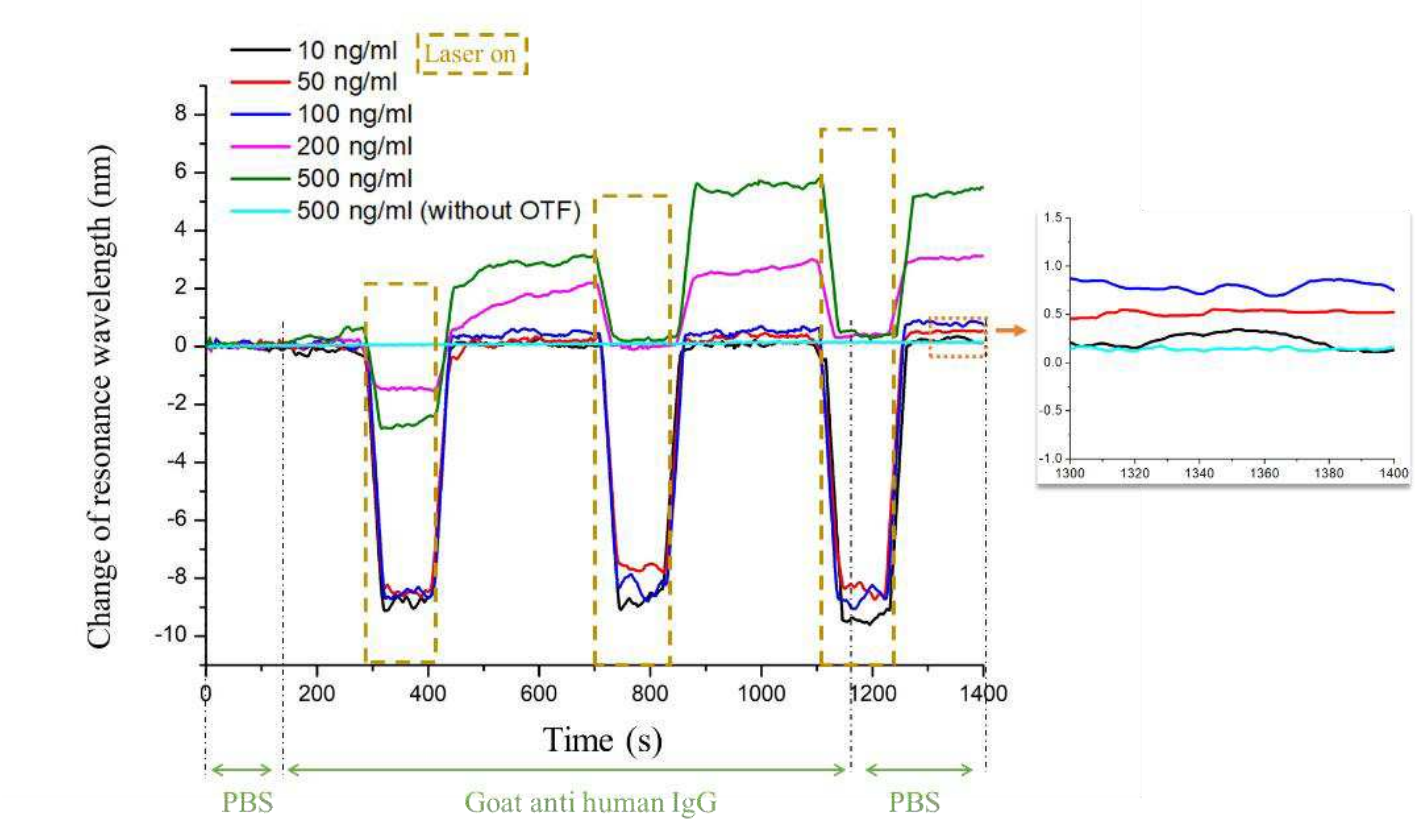

Fig. 6. The enrichment ability test for different Goat anti human IgG concentrations. The change of resonance wavelength is $0.23 \mathrm{~nm}, 0.51 \mathrm{~nm}, 0.78 \mathrm{~nm}, 3.07 \mathrm{~nm}$ and $5.53 \mathrm{~nm}$ for $10 \mathrm{ng} / \mathrm{ml}, 50 \mathrm{ng} / \mathrm{ml}$, $100 \mathrm{ng} / \mathrm{ml}, 200 \mathrm{ng} / \mathrm{ml}$, and $500 \mathrm{ng} / \mathrm{ml}$ with OTF enhancement, and $0.15 \mathrm{~nm}$ for $500 \mathrm{ng} / \mathrm{ml}$ without OTF enhancement. The OTF enhances signal by a factor of 36.9. The calculated lowest concentration of biomolecule is estimated to be $62.5 \mathrm{pM}(10 \mathrm{ng} / \mathrm{ml}$, molecular weight of Goat anti human IgG is $\sim 160 \mathrm{kDa}$ ).

\section{Discussion}

To tackle the difficulty of diffusion-limited biosensing, we developed, through temporal modulation of the thermophoretic force and the convective flow, an OTF method for surface-based in situ protein-protein interaction enhancement. The presented OTF method is a milestone that provides multiple perspectives of using time-varying optothermal phenomena in the biomolecular manipulation and biosensing sensitivity enhancement. The all-optical approach can operate in PBS solution which is most commonly used buffer solution, so the method can be readily adopted in a wide range of biochemical studies. It functionalizes in two steps: First is the enrichment of the low concentration biomolecules to a ring-shaped aggregation, and the second step is to push the biomolecular aggregation closer to their counterparts on the Au film for enhancing the surface capture. This operation continues repeatedly until the highest interaction rate is achieved. It enables the enrichment of nanometer-scale biomolecules at a low-temperature ring-shaped region that situates several micrometers away from the hottest laser spot center. Yet more importantly, the major biomolecular interaction starts at the very moment that heating laser is turned off and 
the heat dissipated rapidly in several microseconds. Thus, the biomolecule interaction is free from the possible thermal damage.

Moreover, the real-time imaging ability of surface plasmon resonance imaging (WSPRi) makes it easy to locate the most responsive region of the biomolecular interactions, and one can randomly choose and manipulate the region of interest for sensing. Thus, we have achieved an enhancement factor of 36.9. This, to our knowledge, is the highest enhancement factor that has ever been achieved in existing active SPR sensing schemes. This is also the first time that the dynamic transient heating dissipation process is adopted for biomolecular manipulation in lab-on-a-chip platform. In addition, OTF is a promising tool for biological sensing of analytes at low levels. It can concentrate biological molecules at picomolar concentrations within a few minutes, and it can be applied to a wide range of biomolecular manipulations since most of them have positive $S_{T}$. Furthermore, the method can potentially obtain a further improved sensitivity and detection limit if it is applied on other sensing platforms or carrying out biomolecular manipulations of other kinds. In conclusion, the present work opens a new epoch in widespread applications in microfluidic biosensing platform when ultrahigh sensitivity is necessary. We believe that it can also promote the development of a wider range of scientific and clinical applications in biological studies.

\section{Methods}

\section{Sensing surface fabrication}

The sensing chip substrate is made of $18 \mathrm{~mm} \times 18 \mathrm{~mm}$ SF11 glass. First a $2 \mathrm{~nm} \mathrm{Cr}$ adhesive layer was coated, and a $48 \mathrm{~nm}$ gold sensing layer successively coated on the substrate via magnetron sputtering. In addition, the prism is an equilateral triangle shape in side view; it is also made by SF11 glass. Refractive index matching oil $(n=1.78)$ was used to exhaust the air between the prism and the back side of the sensing chip. The microfluidic reaction chamber is cylindrical in shape and made of polydimethylsiloxane (PDMS); and its height and radius are $2 \mathrm{~mm}$ and $4 \mathrm{~mm}$, respectively.

\section{Pretreatment of the microfluidic sensing surface}

The microfluidic sensing chip was composed of a lab-made PDMS flow cell and functionalized $\mathrm{Au}$ film. The flow cell and Au film were tightly sealed. (1) The sensing chip was cleaned with DI water, then it was incubated in $10 \mathrm{mM}$ 11-mercaptoundecanoic acid (MUA) for $24 \mathrm{~h}$ to form a selfassembled monolayer on the gold film. (2) Next, we placed the modified gold film on the prism with matching oil for subsequent experiments. Activation of the carboxyl groups was achieved by flow cell infusion of $0.4 \mathrm{~mol} / \mathrm{L}$ 1-(3-Dimethylaminopropyl)-3-ethylcarbodiimide hydrochloride and $0.1 \mathrm{~mol} / \mathrm{L} \mathrm{N}$-Hydroxy succinimide in $0.1 \mathrm{~mol} / \mathrm{L} \mathrm{2-2-( \textrm {N } - M o r p h o l i n o )}$ ethanesulfonic acid sodium salt $\mathrm{pH}=5.5)$. (3) Then the human $\mathrm{lgG}(10 \mu \mathrm{g} / \mathrm{ml})$ in $10 \mathrm{mM}$ acetate $(\mathrm{pH}=4.5)$ was injected into the flow cell for probe immobilization on Au film. (4) After the process of immobilization of human IgG, bovine serum albumin (BSA, $100 \mu \mathrm{g} / \mathrm{ml}$ ) was injected to block the nonspecific adsorption sites. Note that PBS was injected to flush the channel for 10 min after each step.

\section{Near-field temperature mapping}

Temperature measurement was based on the SPR wavelength response to the sample temperature. ${ }^{74}$ The temperature result is a near-field temperature profile under heating laser excitation. Since the resonance wavelength was an indicator of the refractive index ( $n$ ) of the sample in the near-field region which was governed by the Fresnel function, ${ }^{75}$ and the sample's RI is a temperature correlation function $n(T)$, one can choose a sample with a known $n(T)$ as a temperature indicator. Here, we chose $\mathrm{NaCl}$ sodium chloride solution with a concentration of $5 \%$ 
$(0.85 \mathrm{M})$ as the temperature indicator. The solution's $n(T)=a+b T+c T^{2}$, and the corresponding coefficients are $a=1.22775, b=0.000915218$ and $c=-1.69995 \times 10^{-6} .72$

We acquired the resonance wavelength image of the sensing surface by heating laser illumination in room temperature (293K), then translated the resonance wavelength into temperature according to $n(T)$. After temperature calibration, as shown in Fig. 2(b), the temperature profile followed the Gaussian distribution of the laser spot. Therefore, the temperature increment in the ring-shaped enrichment region in our OTF-enhanced biosensing scheme was under $20 \mathrm{~K}$. In addition, the near-field temperature mapping ability of our system was also verified.

\section{Finite element simulation}

The microfluidic chip applied in our experiments is cylindrical in shape. Because it has a rotationally symmetric shape, a 2D model was established to solve the heat transfer and fluid dynamic problem by using the finite-element solver COMSOL Multiphysics. The microfluidic chamber was set exactly at the size of the microfluidic reaction chamber (height $=2 \mathrm{~mm}$, radius $=4 \mathrm{~mm}$ ). The Multiphysics process was studied using Laminar Flow Module and Heat transfer in fluid Module. The initial temperature (room temperature) was set to 293.15K; we mimicked the heating source spot with a radius of $1.5 \mu \mathrm{m}$ in the Gaussian distribution. The material parameters such as the thermal conductivity and heat capacity of glass, gold and water were adapted from the COMSOL material library. The thin layer approximation was adopted to simulate the $48 \mathrm{~nm}$ Au film that coated the glass slide. The total heating power was set to $60 \mathrm{~mW}$. The thermal absorption rate of the $48 \mathrm{~nm} \mathrm{Au}$ film was set as 0.09 , taking the measured optical attenuation rate of the PDMS microfluidic chamber into consideration.

\section{Acknowledgments}

This work was supported by the Project from National Natural Science Foundation of China (61905145, 61775148, and 61527827); National Key Research and Development Program of China (2017YFB0403804); Guangdong Natural Science Foundation and Province Project (2018A030310544); Shenzhen Science and Technology R\&D and Innovation Foundation (JCYJ20180305124754860, JCYJ20200109105608771).

\section{Author contributions}

J. C., Y. Z. and Y. S. conceived the idea and designed the experimental setup; J. Z., Y. Z., and R. M. prepared the bio-samples and did the data processing; X. W. and J. C. did the simulation; J. C., Y. Z., X. W., T. Z., and W. S., did the experiments and analyzed the data; H. Q. and Y. W. analyzed the data of bio-chemical experiments, and all authors discussed the results. J. C., Y. Z., and Y. S. wrote the paper and J. Q., H. P. H., Y. G., and B. G. reviewed and edited the final version. Y. S. supervised the research. All co-authors commented critically on the manuscript.

\section{Competing interests}

The authors declare no competing financial interests. 


\section{Materials \& Correspondence}

Correspondence and requests for materials should be addressed to J.C. or Y. S.

\section{Data availability}

The data that support the findings of this study are available from the corresponding authors upon reasonable request.

\section{References}

1 Wang, J. Electrochemical biosensors: towards point-of-care cancer diagnostics. Biosensors and Bioelectronics 21, 1887-1892 (2006).

2 Brangel, P. et al. A serological point-of-care test for the detection of IgG antibodies against Ebola virus in human survivors. ACS nano 12, 63-73 (2018).

3 Ju, H., Yan, G., Chen, F. \& Chen, H. Enzyme - Linked Immunoassay of a - 1 - Fetoprotein in Serum by Differential Pulse Voltammetry. Electroanalysis: An International Journal Devoted to Fundamental and Practical Aspects of Electroanalysis 11, 124-128 (1999).

4 Sun, W., Jiao, K., Zhang, S., Zhang, C. \& Zhang, Z. Electrochemical detection for horseradish peroxidase-based enzyme immunoassay using $\mathrm{p}$-aminophenol as substrate and its application in detection of plant virus. Analytica chimica acta 434, 43-50 (2001).

5 Lequin, R. M. Enzyme immunoassay (EIA)/enzyme-linked immunosorbent assay (ELISA). Clinical chemistry 51, 2415-2418 (2005).

6 Song, Y., Wei, W. \& Qu, X. Colorimetric biosensing using smart materials. Advanced Materials 23, 4215-4236 (2011).

7 Zhang, C., Yuan, Y., Zhang, S., Wang, Y. \& Liu, Z. Biosensing platform based on fluorescence resonance energy transfer from upconverting nanocrystals to graphene oxide. Angewandte Chemie International Edition 50, 6851-6854 (2011).

Li, J. \& Zhu, J.-J. Quantum dots for fluorescent biosensing and bio-imaging applications. Analyst 138, 2506-2515 (2013).

9 Zhong, W. Nanomaterials in fluorescence-based biosensing. Analytical and Bioanalytical Chemistry 394, 47-59 (2009).

10 Dufour, D. R., Talastas, M., Fernandez, M. D. \& Harris, B. Chemiluminescence assay improves specificity of hepatitis C antibody detection. Clinical chemistry 49, 940-944 (2003).

11 Tanaka, T. \& Matsunaga, T. Fully automated chemiluminescence immunoassay of insulin using antibody- protein A- bacterial magnetic particle complexes. Analytical chemistry 72, 35183522 (2000).

12 Laing, S., Jamieson, L. E., Faulds, K. \& Graham, D. Surface-enhanced Raman spectroscopy for in vivo biosensing. Nature Reviews Chemistry 1, 1-19 (2017).

13 Henry, A.-I., Sharma, B., Cardinal, M. F., Kurouski, D. \& Van Duyne, R. P. Surface-enhanced Raman spectroscopy biosensing: in vivo diagnostics and multimodal imaging. Analytical chemistry 88, 6638-6647 (2016).

14 Chou, I.-H. et al. Nanofluidic biosensing for $\beta$-amyloid detection using surface enhanced Raman spectroscopy. Nano letters 8, 1729-1735 (2008). 
Tripp, R. A., Dluhy, R. A. \& Zhao, Y. Novel nanostructures for SERS biosensing. Nano Today 3, 31-37 (2008)

Mejía-Salazar, J. \& Oliveira Jr, O. N. Plasmonic biosensing: focus review. Chemical reviews 118, 10617-10625 (2018).

Law, W.-C., Yong, K.-T., Baev, A., Hu, R. \& Prasad, P. N. Nanoparticle enhanced surface plasmon resonance biosensing: application of gold nanorods. Optics express 17, 19041-19046 (2009).

Lynn Jr, N. S. et al. Biosensing enhancement using passive mixing structures for microarraybased sensors. Biosensors and Bioelectronics 54, 506-514 (2014).

Chen, Z.-H. et al. Sharp convex gold grooves for fluorescence enhancement in micro/nano fluidic biosensing. Journal of Materials Chemistry B 5, 8839-8844 (2017).

De Angelis, F. et al. Breaking the diffusion limit with super-hydrophobic delivery of molecules to plasmonic nanofocusing SERS structures. Nature Photonics 5, 682-687 (2011).

Liu, R. H., Lenigk, R., Druyor-Sanchez, R. L., Yang, J. \& Grodzinski, P. Hybridization enhancement using cavitation microstreaming. Analytical Chemistry 75, 1911-1917 (2003).

Voiculescu, I. \& Nordin, A. N. Acoustic wave based MEMS devices for biosensing applications. Biosensors and Bioelectronics 33, 1-9 (2012).

Ozcelik, A. et al. Acoustic tweezers for the life sciences. Nature methods 15, 1021-1028 (2018). Liu, X. et al. Development of an AC electrokinetics-based immunoassay system for on-site serodiagnosis of infectious diseases. Sensors and Actuators A: Physical 171, 406-413 (2011).

Barik, A. et al. Graphene-edge dielectrophoretic tweezers for trapping of biomolecules. Nature communications 8, 1867 (2017).

Li, H. et al. Chemical and biomolecule sensing with organic field-effect transistors. Chemical reviews 119, 3-35 (2018).

Kim, Y., Ding, H. \& Zheng, Y. Enhancing surface capture and sensing of proteins with low-power optothermal bubbles in a biphasic liquid. Nano Letters 20, 7020-7027 (2020).

Fränzl, M. et al. Thermophoretic trap for single amyloid fibril and protein aggregation studies. Nature Methods 16, 611-614 (2019).

Garcia-Guirado, J. et al. Overcoming diffusion-limited biosensing by electrothermoplasmonics. ACS Photonics 5, 3673-3679 (2018).

Hong, C., Yang, S. \& Ndukaife, J. C. Stand-off trapping and manipulation of sub-10 nm objects and biomolecules using opto-thermo-electrohydrodynamic tweezers. Nature Nanotechnology 15, 908-913 (2020).

Karim, F., Vasquez, E. S., Sun, Y. \& Zhao, C. Optothermal microbubble assisted manufacturing of nanogap-rich structures for active chemical sensing. Nanoscale 11, 20589-20597 (2019).

(1)

Zhou, J. et al. The capture of antibodies by antibody-binding proteins for ABO blood typing using SPR imaging-based sensing technology. Sensors and Actuators B: Chemical 304, 127391 (2020).

Sereda, A., Moreau, J., Canva, M. \& Maillart, E. High performance multi-spectral interrogation for surface plasmon resonance imaging sensors. Biosensors and Bioelectronics 54, 175-180 (2014).

Yuk, J. S. et al. Analysis of protein interactions on protein arrays by a wavelength interrogation - based surface plasmon resonance biosensor. Proteomics 4, 3468-3476 (2004). Wang, X. et al. Ultrafast Surface Plasmon Resonance Imaging Sensor via the High-Precision Four-Parameter-Based Spectral Curve Readjusting Method. Analytical Chemistry (2020). 
Zeng, Y. et al. Recent advances in surface plasmon resonance imaging: detection speed, sensitivity, and portability. Nanophotonics 6, 1017-1030 (2017).

Zeng, Y., Zhou, J., Wang, X., Cai, Z. \& Shao, Y. Wavelength-scanning surface plasmon resonance microscopy: A novel tool for real time sensing of cell-substrate interactions. Biosensors and Bioelectronics 145, 111717 (2019).

Liedberg, B., Lundström, I. \& Stenberg, E. Principles of biosensing with an extended coupling matrix and surface plasmon resonance. Sensors and Actuators B: Chemical 11, 63-72 (1993). Wu, L., Guo, J., Dai, X., Xiang, Y. \& Fan, D. Sensitivity enhanced by MoS 2-graphene hybrid structure in guided-wave surface plasmon resonance biosensor. Plasmonics 13, 281-285 (2018). Chen, K. et al. Fast spectral surface plasmon resonance imaging sensor for real-time highthroughput detection of biomolecular interactions. Journal of biomedical optics 21, 127003 (2016).

Zeng, Y. et al. High-throughput imaging surface plasmon resonance biosensing based on ultrafast two-point spectral-dip tracking scheme. Optics Express 28, 20624-20633 (2020). Zeng, Y. et al. High-throughput imaging surface plasmon resonance biosensing based on an adaptive spectral-dip tracking scheme. Optics express 24, 28303-28311 (2016). Eslahian, K. A., Majee, A., Maskos, M. \& Würger, A. Specific salt effects on thermophoresis of charged colloids. Soft Matter 10, 1931-1936 (2014). Lüsebrink, D., Yang, M. \& Ripoll, M. Thermophoresis of colloids by mesoscale simulations. Journal of Physics: Condensed Matter 24, 284132 (2012). Vigolo, D., Buzzaccaro, S. \& Piazza, R. Thermophoresis and thermoelectricity in surfactant solutions. Langmuir 26, 7792-7801 (2010).

Jiang, Q., Rogez, B., Claude, J.-B., Baffou, G. \& Wenger, J. Quantifying the Role of the Surfactant and the Thermophoretic Force in Plasmonic Nano-optical Trapping. Nano Letters 20, 88118817 (2020).

Reichl, M., Herzog, M., Götz, A. \& Braun, D. Why charged molecules move across a temperature gradient: the role of electric fields. Physical Review Letters 112, 198101 (2014).

Cong, H., Chen, J. \& Ho, H.-P. Trapping, sorting and transferring of micro-particles and live cells using electric current-induced thermal tweezers. Sensors and Actuators B: Chemical 264, 224233 (2018).

Ding, H., Kollipara, P. S., Lin, L. \& Zheng, Y. Atomistic modeling and rational design of optothermal tweezers for targeted applications. Nano Research 14, 295-303 (2021). Chen, J. et al. Thermal gradient induced tweezers for the manipulation of particles and cells. Scientific reports 6, 1-13 (2016). Braun, M. \& Cichos, F. Optically controlled thermophoretic trapping of single nano-objects. ACS nano 7, 11200-11208 (2013). Lin, L. et al. Opto-thermoelectric nanotweezers. Nature photonics 12, 195-201 (2018). Baffou, G., Quidant, R. \& García de Abajo, F. J. Nanoscale control of optical heating in complex plasmonic systems. ACS nano 4, 709-716 (2010).

Xin, H. et al. Optical forces: from fundamental to biological applications. Advanced Materials 32, 2001994 (2020). Kang, Z. et al. Trapping and assembling of particles and live cells on large-scale random gold nano-island substrates. Scientific Reports 5, doi:10.1038/srep09978 (2015). 
trapping live cells and colloidal particles. Optics letters 40, 3926-3929 (2015).

Chen, J. et al. Thermal optofluidics: principles and applications. Advanced Optical Materials 8 , 1900829 (2020).

Azimzadeh, A. \& Van Regenmortel, M. Antibody affinity measurements. Journal of Molecular Recognition 3, 108-116 (1990).

Donner, J. S., Baffou, G., McCloskey, D. \& Quidant, R. Plasmon-assisted optofluidics. Acs nano 5, 5457-5462 (2011).

60 Braibanti, M., Vigolo, D. \& Piazza, R. Does thermophoretic mobility depend on particle size? Physical review letters 100, 108303 (2008).

Putnam, S. A. \& Cahill, D. G. Transport of nanoscale latex spheres in a temperature gradient. Langmuir 21, 5317-5323 (2005).

Putnam, S. A., Cahill, D. G. \& Wong, G. C. Temperature dependence of thermodiffusion in aqueous suspensions of charged nanoparticles. Langmuir 23, 9221-9228 (2007).

Würger, A. Transport in charged colloids driven by thermoelectricity. Physical review letters 101, 108302 (2008).

Rasuli, S. N. \& Golestanian, R. Soret motion of a charged spherical colloid. Physical review letters 101, 108301 (2008).

Ding, H., Kollipara, P. S., Lin, L. \& Zheng, Y. Atomistic modeling and rational design of optothermal tweezers for targeted applications. Nano Research 14, 295-303 (2020).

Filoti, D. I., Shire, S. J., Yadav, S. \& Laue, T. M. Comparative study of analytical techniques for determining protein charge. Journal of Pharmaceutical Sciences 104, 2123-2131 (2015).

Yang, D., Kroe-Barrett, R., Singh, S. \& Laue, T. IgG charge: practical and biological implications. Antibodies 8, 24 (2019).

Braun, D. \& Libchaber, A. Trapping of DNA by thermophoretic depletion and convection. Physical review letters 89, 188103 (2002).

Hatlo, M. M. \& Lue, L. The role of image charges in the interactions between colloidal particles. Soft Matter 4, 1582-1596 (2008).

Schein, P., Kang, P., O'Dell, D. \& Erickson, D. Nanophotonic force microscopy: characterizing particle-surface interactions using near-field photonics. Nano letters 15, 1414-1420 (2015).

Ng, S. P., Wu, C. M. L., Wu, S. Y. \& Ho, H. P. White-light spectral interferometry for surface plasmon resonance sensing applications. Optics Express 19, 4521-4527 (2011).

Aly, K. M. \& Esmail, E. Refractive index of salt water: effect of temperature. Optical materials 2, 195-199 (1993).

Law, W.-C., Yong, K.-T., Baev, A. \& Prasad, P. N. Sensitivity improved surface plasmon resonance biosensor for cancer biomarker detection based on plasmonic enhancement. ACS nano 5, 4858-4864 (2011).

Kim, I. T. \& Kihm, K. D. Full-field and real-time surface plasmon resonance imaging thermometry. Optics letters 32, 3456-3458 (2007).

Kretschmann, E. Die bestimmung optischer konstanten von metallen durch anregung von oberflächenplasmaschwingungen. Zeitschrift für Physik A Hadrons and nuclei 241, 313-324 (1971). 


\section{Figures}

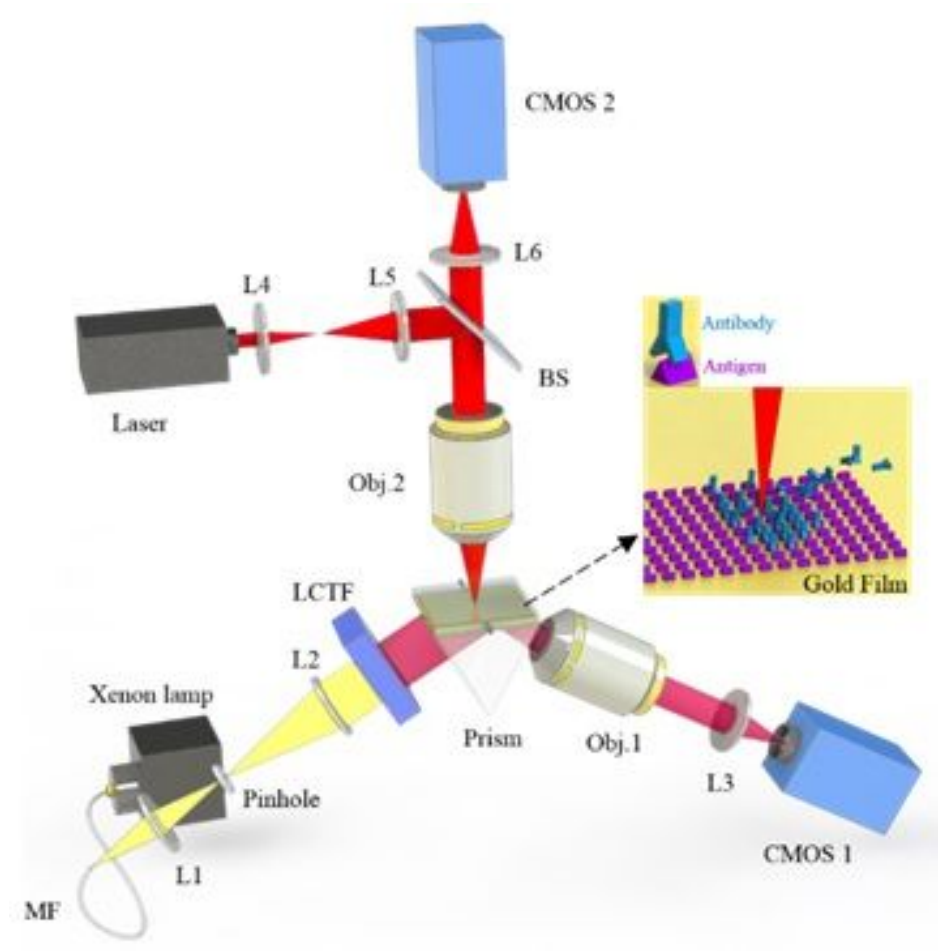

\section{Figure 1}

The optical setup. The system consisted of two parts: WSPRi path and optothermal excitation path. L1L6: Lens; Obj.1-2: Objects lens; MF: Multimode optical fiber; BS: Beam splitting.
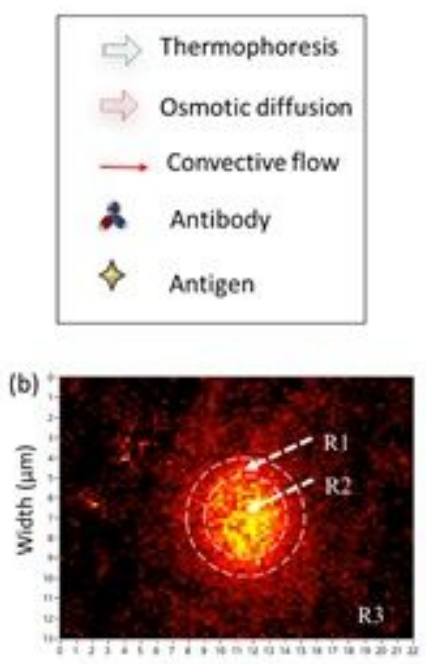

Length (um)
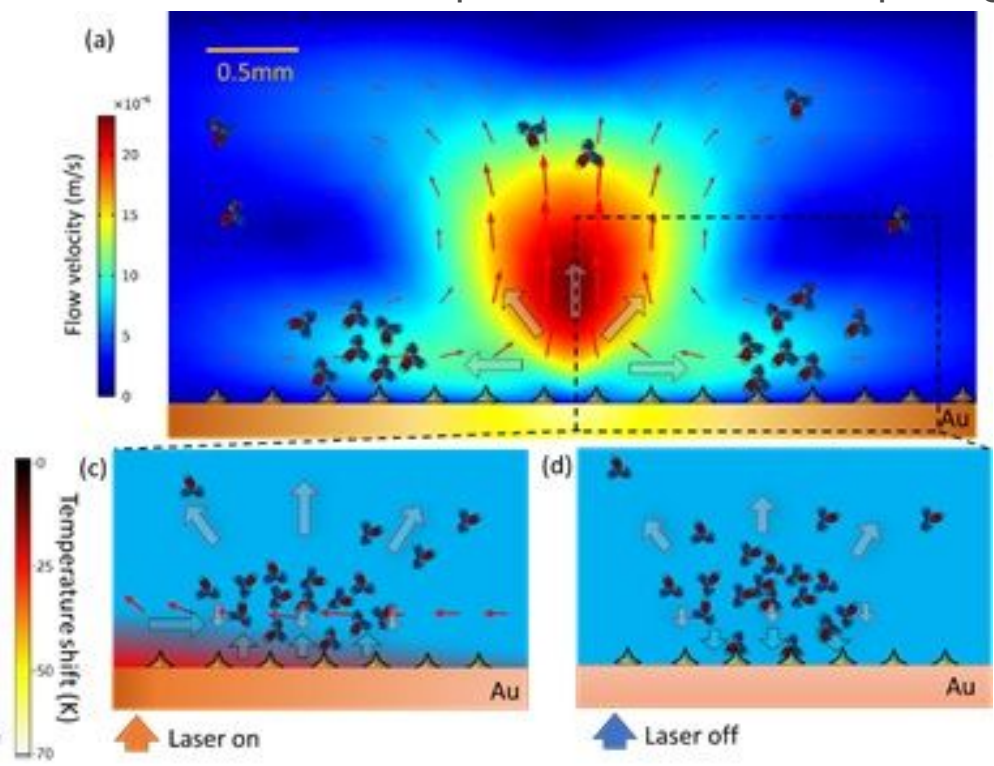

\section{Figure 2}

Schematic illustration and theoretical analysis of OTF system. (a) Side view of the entire system when the heating laser is switching on. The convective flow and thermophoresis relocated and enriched the 
biomolecules from bulk solution to a ring shape region where the temperature is in relatively lower level; (b) Top view of the ring shape biomolecule aggregation region in the background of near-field temperature map. R1: Biomolecule enrichment and binding region; R2: heating laser focusing center region; R3: background region with no laser heating. (c) Zoom-in picture of the biomolecule enrichment region when laser is switched on, the upward thermophoretic (UT) force induces inefficient binding of the biomolecules; (d) Zoom-in picture of the biomolecule enrichment region when laser is just switched off, the flipped thermophoretic (FT) force (downward) induces efficient binding of the biomolecules.

(a)

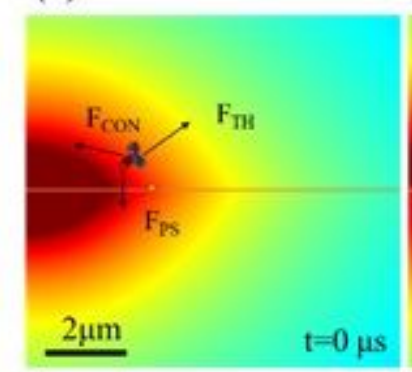

(d)

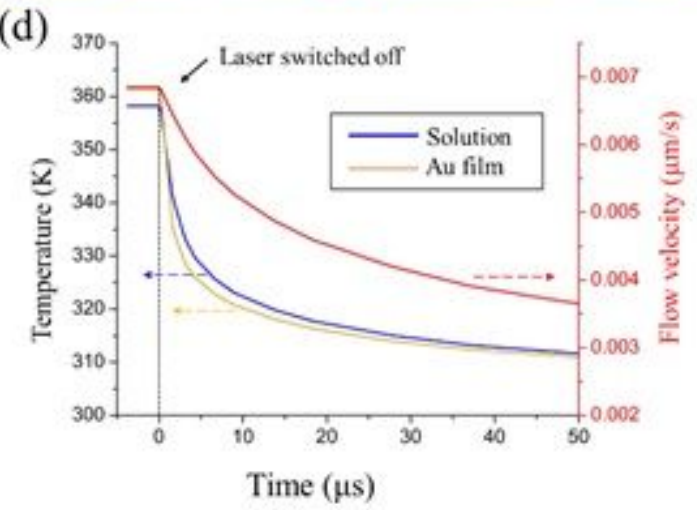

(c)

(b)

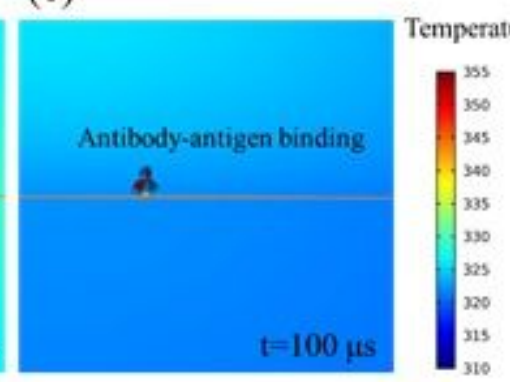

(e)

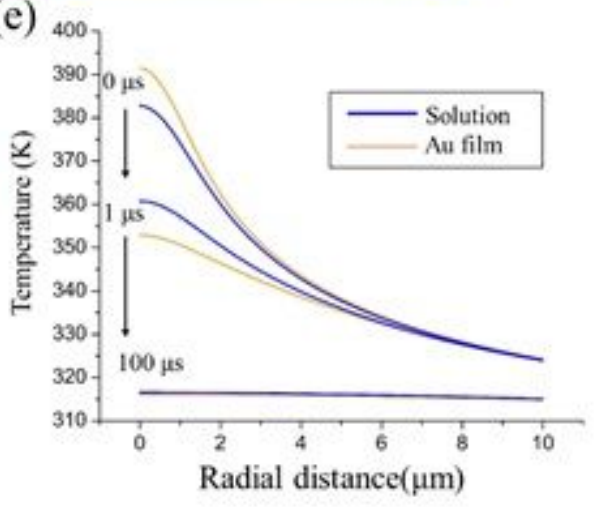

\section{Figure 3}

Temperature distribution and force analysis in OTF antibody-antigen binding enhancement method. (a)-

(c) Temperature distribution (symmetrical half) near the laser spot at $0 \mu \mathrm{s}$ (heating laser switched off), $1 \mu \mathrm{s}$, and $100 \mu \mathrm{s}$. F_CON,F_TH, and F_PS are convective drag force, thermophoretic force, and particlesurface interaction force respectively. (d) Temperature temporal variation comparison between the sample solution and the Au film surface as well as the convective flow velocity variation. The temperature and flow velocity interrogation point locate at $1.5 \mu \mathrm{m}$ radial distance from the laser spot center and 200 $\mathrm{nm}$ above the Au film. (e) Temperature radially distribution in $200 \mathrm{~nm}$ above the Au film. 
(a)

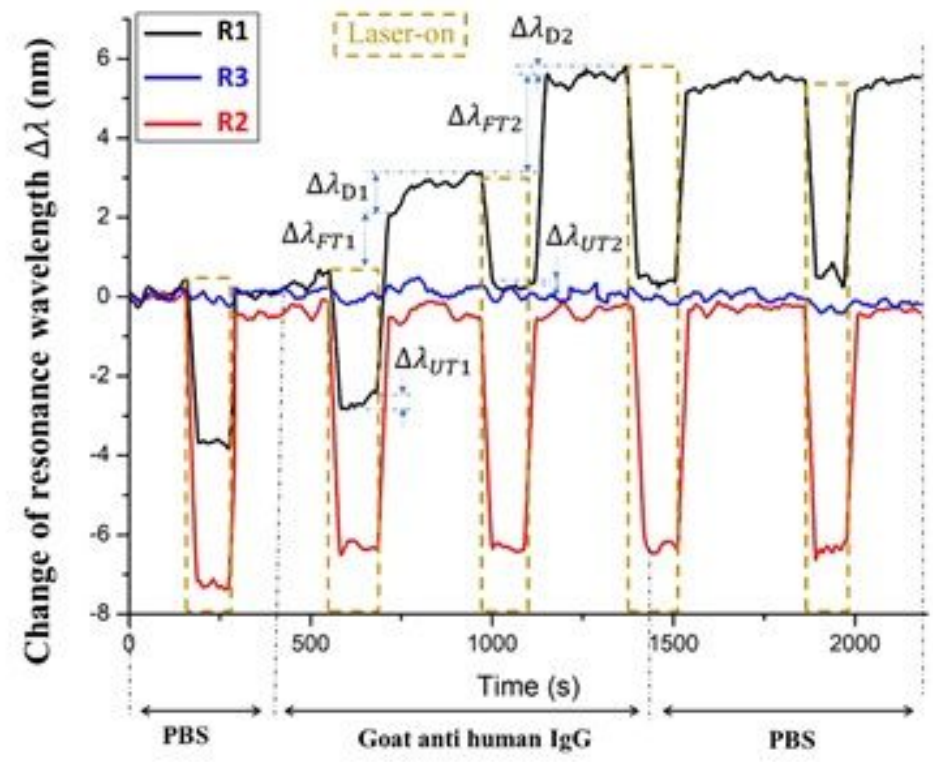

(b)
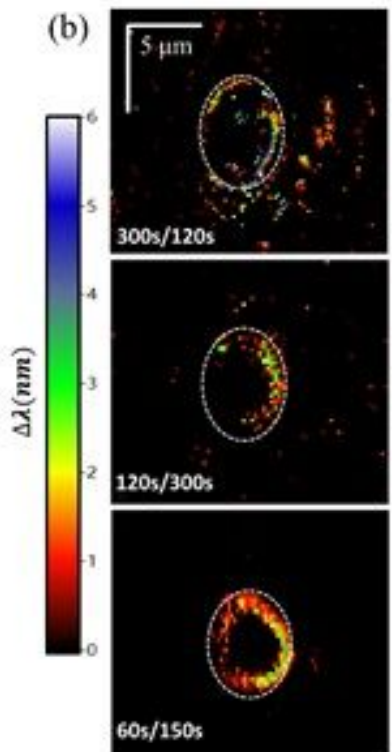

\section{Figure 4}

Experimental demonstration of OTF-enhanced SPR imaging capabilities via binding of $0.5 \mu \mathrm{g} / \mathrm{mL}$ goat anti human IgG with the probes on gold surface. (a) Temporal variation of the $\Delta \lambda$ induced by the heating laser on/off modulation (120s/300s on/off period). Three curves are the resonance wavelength real-time response curves, and each point on the curve is the averaged value in the corresponding sub-regions $(4 \times 6$ pixels) that produce the largest resonance wavelength shift from the three interrogation regions of R1, R2 and R3 in IgG binding experiments. (b) SPR image of the whole sensing site of three on/off period of $300 \mathrm{~s} / 120 \mathrm{~s}, 120 \mathrm{~s} / 300 \mathrm{~s}$, and $60 \mathrm{~s} / 120 \mathrm{~s}$, respectively. The change of resonance wavelength $\Delta \lambda$ indicates the SPR peak shifting after the experiment. 
(a)

$\mathrm{T}=420 \mathrm{~s}$

$\mathrm{T}=420 \mathrm{~s}$

$\prod_{120.0 \mathrm{ff}}^{100, \text { on }} \square \square \square$
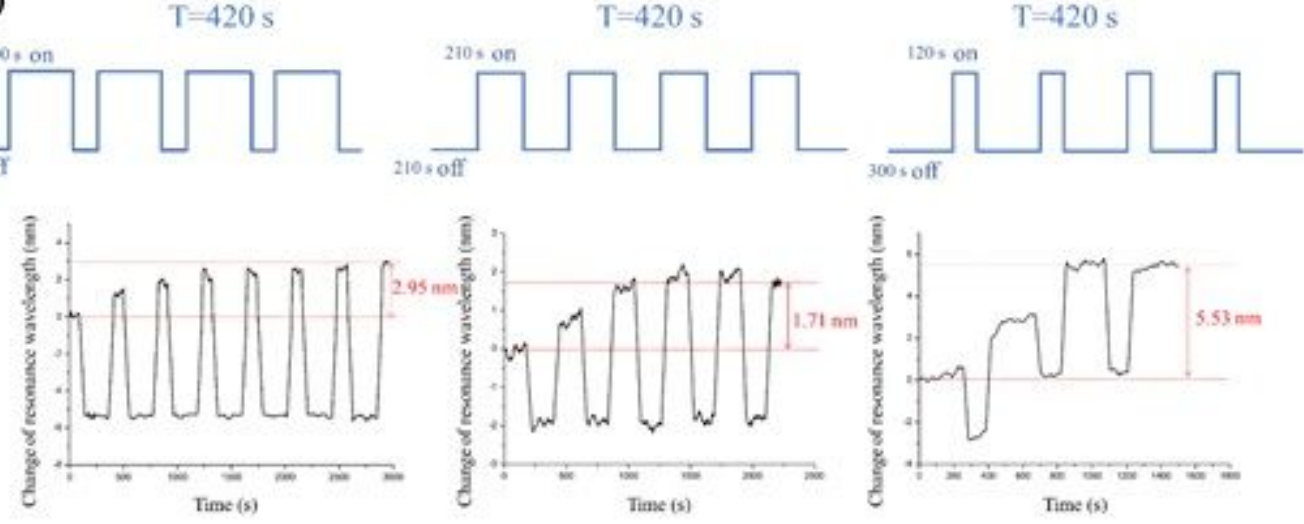

(b)
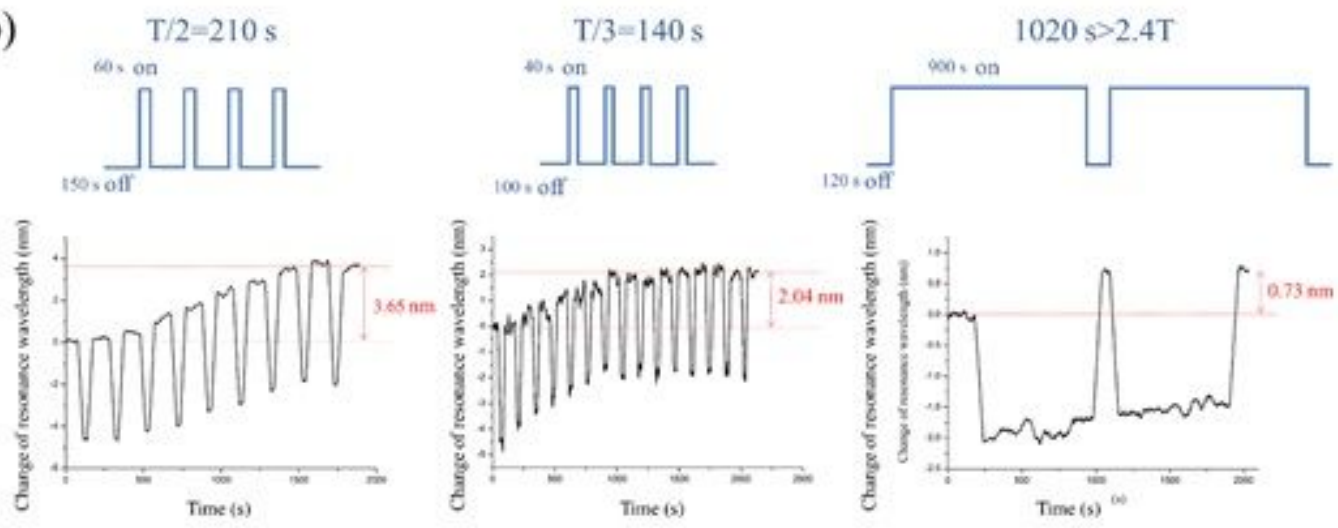

\section{Figure 5}

Optimized heating laser on/off time investigation in goat anti human IgG $(500 \mathrm{~nm} / \mathrm{ml})$. Under the heating laser power of $60 \mathrm{~mW}$, a series of heating laser triggering signal (laser on/off duration) of different (a) duty cycles and (b) period T are studied. The net SPR wavelength shift after the experiments $\Delta \lambda$ is denoted in red. Each point on the curves is the averaged value of resonance wavelength change in the corresponding sub-regions of $4 \times 6$ pixels that produce the largest $\Delta \lambda$ from the ring-shape interrogation region R1. 


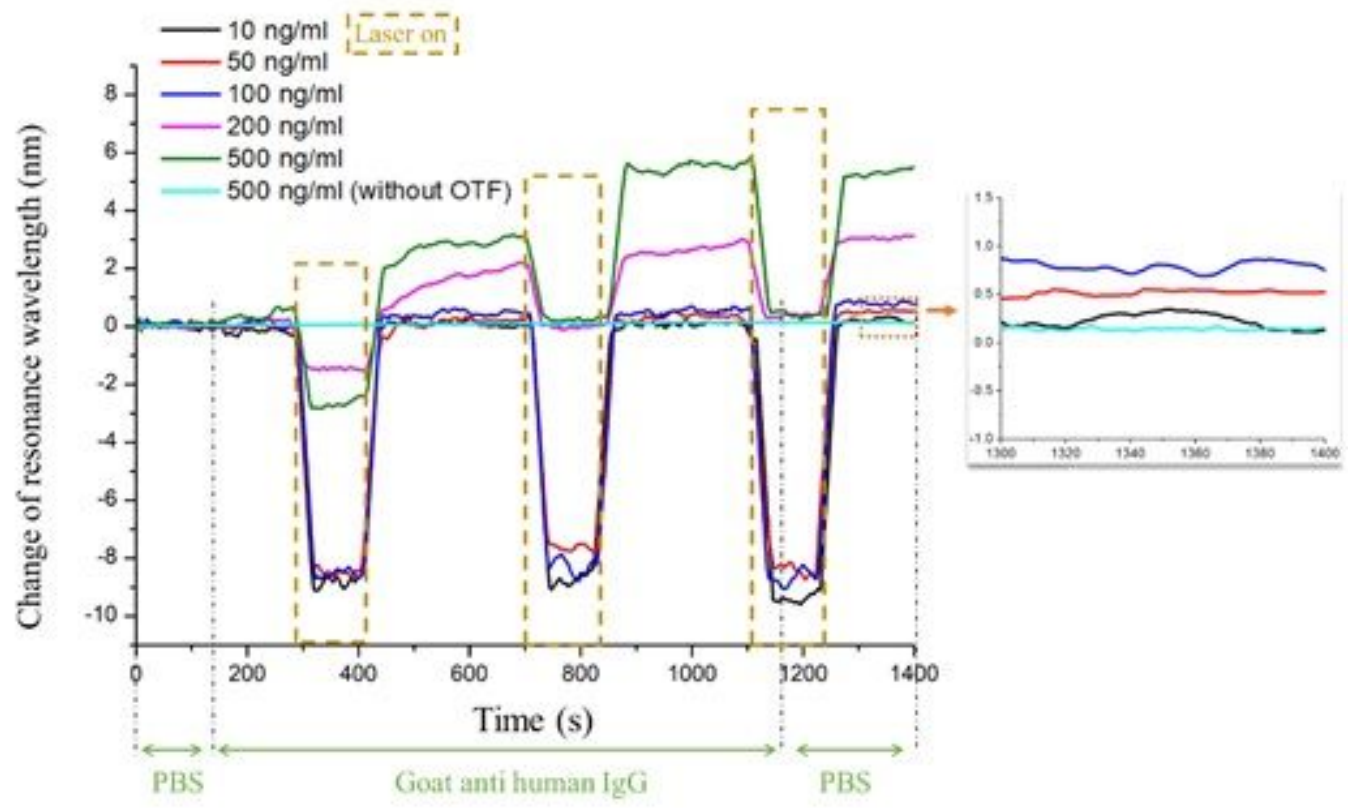

Figure 6

The enrichment ability test for different Goat anti human IgG concentrations. The change of resonance wavelength is $0.23 \mathrm{~nm}, 0.51 \mathrm{~nm}, 0.78 \mathrm{~nm}, 3.07 \mathrm{~nm}$ and $5.53 \mathrm{~nm}$ for $10 \mathrm{ng} / \mathrm{ml}, 50 \mathrm{ng} / \mathrm{ml}, 100 \mathrm{ng} / \mathrm{ml}, 200$ $\mathrm{ng} / \mathrm{ml}$, and $500 \mathrm{ng} / \mathrm{ml}$ with OTF enhancement, and $0.15 \mathrm{~nm}$ for $500 \mathrm{ng} / \mathrm{ml}$ without OTF enhancement. The OTF enhances signal by a factor of 36.9. The calculated lowest concentration of biomolecule is estimated to be $62.5 \mathrm{pM}(10 \mathrm{ng} / \mathrm{ml}$, molecular weight of Goat anti human IgG is $\otimes 160 \mathrm{kDa})$. 